\title{
Brasilien als internationaler Akteur
}

\author{
Peter Birle
}

\section{Einleitung}

Brasilien ist seit Mitte der 1990er Jahre in zunehmendem Maße als globaler politischer Akteur in Erscheinung getreten. Unter den Präsidenten Fernando Henrique Cardoso (1995-2002) und Luiz Inácio Lula da Silva (2003-2010) sowie seit 2011 unter Präsidentin Dilma Rousseff hat das Land nicht nur in Lateinamerika verstärkt politische Verantwortung übernommen und zahlreiche Anstöße für neue regionale Kooperations- und Integrationsprozesse gegeben, sondern sich auch über die eigene Region hinaus profiliert: durch eine aktive Afrikapolitik, durch die Mitarbeit in multilateralen Süd-Süd-Foren wie der BRICS-Gruppe (Brasilien, Russland, Indien, China und Südafrika) und dem IBSA-Forum (Indien, Brasilien, Südafrika), durch eine prominente Rolle bei den Diskussionen über die Zukunft der Welthandelsordnung und durch Vermittlungsangebote in internationalen Konflikten. Die brasilianische Außenpolitik zeichnet sich dabei gleichermaßen durch Kontinuitäten und Brüche aus. Während das außerordentlich aktive Engagement neu ist, bestehen viele Grundprinzipien der internationalen Einbindung Brasiliens schon seit Beginn des 20. Jahrhunderts. Der folgende Beitrag beschreibt zunächst einige Konstanten und Grundprinzipien der brasilianischen Außenpolitik und geht dann auf die internen und externen Faktoren ein, die in den vergangenen zwei Jahrzehnten den Aufstieg des Landes zu einem global player ermöglicht haben. Im Anschluss daran erfolgt ein Blick auf die Beziehungen mit Lateinamerika, auf die Süd-Süd-Politik sowie auf die Politik gegenüber den USA und Europa.

\section{Kontinuität und Wandel der Außenpolitik}

Mit fast 200 Millionen Einwohnern, einem Territorium von $8.547 .000 \mathrm{~km}^{2}$ und einem Bruttoinlandsprodukt, das zu den zehn größten weltweit gehört, ist Brasilien ein Land von kontinentalen Dimensionen. Es gehört 
zu den grundlegenden Konstanten der brasilianischen Außenpolitik, dass die politischen Eliten aus der Größe des Landes traditionell eine Ambition, gewissermaßen einen natürlichen Anspruch Brasiliens abgeleitet haben, eine wichtige Rolle in der Welt zu spielen. Dieses Statusbewusstsein war bereits vorhanden, lange bevor die Militärs in den 1960er und 1970er Jahren ihre Großmachtträume in geopolitischen Kategorien artikulierten, und es hat auch nach der Rückkehr zur Demokratie seinen Platz im Kanon der außenpolitischen Grundüberzeugungen nicht eingebüßt. Die Gewissheit, dass Brasilien aufgrund seiner Größe ein Platz im Konzert der global players zustehe, verband sich mit der Einsicht, dass es dazu zunächst seine wirtschaftliche Abhängigkeit überwinden und die nationale Entwicklung fördern müsse. Insofern besteht eine zweite zentrale Konstante des auBenpolitischen Denkens in der Vorstellung, dass die internationalen Beziehungen dazu beitragen müssen, die interne wirtschaftliche und soziale Entwicklung voranzutreiben. Umgekehrt hat dieser desarrollistische Grundzug dazu geführt, dass die außenpolitische Agenda stets stark durch das jeweils vorherrschende Wirtschaftsmodell beeinflusst wurde.

Zwei weitere Konstanten der brasilianischen Außenpolitik lauten Universalismus und Autonomie. Brasilien erhebt den Anspruch, nicht nur in Lateinamerika eine wichtige Rolle zu spielen, sondern substantielle Beziehungen zu allen Weltregionen zu pflegen; und es strebt gleichzeitig danach, seine internationale Einbindung frei von Zwängen zu gestalten, die dem Land durch andere Staaten oder durch einseitige Abhängigkeiten auferlegt werden könnten. Während dieses Streben nach Autonomie eine Konstante ist, hat sich die Art und Weise, wie die Autonomie erlangt bzw. erhalten werden soll, in den vergangenen Jahrzehnten verändert. Vigevani/Cepaluni (2010) unterscheiden in dieser Hinsicht zwischen drei grundlegenden Strategien der brasilianischen Außenpolitik:

- Autonomie durch Distanz, d. h. Wahrung der eigenen Souveränität durch die Nichtbeteiligung an internationalen Vereinbarungen;

- Autonomie durch Partizipation, d. h. der Versuch, die internationale Agenda durch eine aktive Beteiligung an internationalen Diskussionen und Vereinbarungen im Sinne der eigenen Interessen zu beeinflussen und zu verändern;

- Autonomie durch Diversifizierung, d. h. die Etablierung von strategischen Partnerschaften mit Ländern, die eine ähnliche Position in der globalen Machthierarchie einnehmen, um so die internationale Ord- 
nung im Sinne der Entwicklungsländer zu verändern, Gegengewichte zu den von den entwickelten Ländern vorgegebenen Prioritäten aufzubauen und die eigene Verhandlungs- und Gestaltungsmacht im internationalen System zu erhöhen.

Keine dieser drei Strategien wird in Reinkultur verfolgt, es handelt sich eher um unterschiedliche Mischungsverhältnisse. Die Regierungen der Streitkräfte (1964-1985) setzten auf defensive Positionen im Hinblick auf internationale Vereinbarungen im Bereich der Menschenrechte, der Kontrolle sensibler Technologien und der Nichtverbreitung von Atomwaffen (Autonomie durch Distanz). Gleichwohl engagierte sich Brasilien schon in den 1970er Jahren aktiv im Rahmen der damaligen Nord-Süd-Agenda und schloss sich der Forderung nach einer Neuen Weltwirtschaftsordnung an. Nach dem Ende der Militärherrschaft nahm die Partizipation in internationalen Foren weiter zu. Die Cardoso-Administration bemühte sich in den 1990er Jahren durch eine aktive Mitarbeit in vielen internationalen Gremien darum, das Erbe der Militärdiktatur hinter sich zu lassen und den Status des Landes zu verbessern. Zu einem zentralen Ziel der brasilianischen Außenpolitik gehört seitdem ein ständiger Sitz in einem reformierten Sicherheitsrat der Vereinten Nationen (VN). Aber während Cardoso vor allem darauf setzte, dass Brasilien in den Club der Mächtigen aufgenommen wird, ohne allzu sehr auf grundlegende strukturelle Veränderungen des internationalen Systems zu pochen, trat die Regierung Lula sehr aktiv für grundlegende Reformen des VN-Systems, der Welthandelsordnung und der internationalen Finanzordnung ein und bediente sich dabei einer Strategie der Autonomie durch Diversifizierung.

Weitere zentrale Merkmale der brasilianischen Außenpolitik sind ihre Professionalität, ihre pragmatische Grundhaltung und ihre langfristige Orientierung im Sinne einer "Staatspolitik". Entscheidende Weichenstellungen in dieser Richtung wurden schon zu Beginn des 20. Jahrhunderts unter Außenminister Baron von Rio Branco (1902-1912) vorgenommen. Rio Branco sorgte für die Institutionalisierung eines professionell arbeitenden Außenministeriums - bekannt als Itamaraty, in Anlehnung an den Namen des Palastes, der von 1899 bis 1970 als Sitz der Behörde diente sowie eines funktionierenden diplomatischen Dienstes. Während Außenpolitik in vielen anderen lateinamerikanischen Ländern vor allem auf Präsidialdiplomatie basiert, wurde in Brasilien das Außenministerium selbst zu einem wichtigen institutionellen Akteur. Das Itamaraty fungiert als in- 
stitutionelles Gedächtnis einer diplomatischen Tradition (Lafer 2001). Die starke Position des Außenministeriums sorgt dafür, dass Regierungswechsel in der Regel nicht zu brüsken Veränderungen der Außenpolitik führen, wie dies beispielsweise im Nachbarland Argentinien in den vergangenen Jahrzehnten immer wieder der Fall gewesen ist.

Gleichwohl ist auch die brasilianische Außenpolitik nicht in Stein gemeißelt. Innerhalb des Außenministeriums existieren unterschiedliche Strömungen, deren jeweiliger Einfluss sich verschieben kann. Während die Befürworter einer starken Süd-Süd-Orientierung unter Präsident Lula an Einfluss gewannen, wurden die "pragmatischen Institutionalisten" zurückgedrängt. Kritiker beklagten unter Lula auch eine "Politisierung" der Außenpolitik. Vor den Präsidentschaftswahlen von 2010 wurde die Außenpolitik erstmals zu einem Wahlkampfthema. Mit der Regierungsübernahme durch Präsidentin Rousseff sind diese Auseinandersetzungen wieder abgeflaut, was damit zusammenhängen dürfte, dass sie einen wesentlich nüchterneren Politikstil vertritt als ihr Vorgänger, der sich im Rahmen unzähliger Auslandsreisen stark persönlich auf internationalem Parkett engagierte und dabei keine Berührungsängste mit umstrittenen Amtskollegen wie dem iranischen Präsidenten Ahmadinedschad zeigte.

Auch die geographischen Prioritäten der Außenpolitik haben sich seit der Regierung Lula verschoben. In einem Aufsatz für die Zeitschrift Revista Brasileira de Politica Internacional zog der damalige Außenminister Celso Amorim im Jahr 2010 eine erste Bilanz zum Ende der Regierung Lula. Allein der Umfang und die Reihenfolge der von ihm angesprochenen Themen der außenpolitischen Agenda sprechen eine deutliche Sprache: Zunächst äußert sich Amorim auf 13,5 Seiten zu den weltpolitischen Veränderungen, zur neuen Rolle Brasiliens, zu Fragen der global governance sowie zu den Themen Solidarität und Universalismus. Es folgen vier Seiten zu Südamerika, eineinhalb Seiten zu den Süd-Süd-Beziehungen allgemein und vier Seiten zu Afrika, dem Mittleren Osten and beyond. Auf gut einer Seite werden danach die Beziehungen mit Europa und den USA kurz abgehandelt (Amorim 2010). Ein Blick in Reden der gegenwärtigen Präsidentin Dilma Rousseff bestätigt diese Prioritätensetzung: Lateinamerika und Afrika stehen an erster Stelle, es folgen die übrigen Süd-SüdBeziehungen, erst danach interessieren die Beziehungen mit den USA und Europa (Rousseff 2013). 


\section{Voraussetzungen für den Aufstieg zum global player}

Brasiliens internationaler Aufstieg im Verlauf der letzten zwei Jahrzehnte war nicht nur das Ergebnis einer bewussten politischen Entscheidung, sondern er basierte auch auf einer Reihe von internen und externen Voraussetzungen. Ohne die innenpolitische und makro-ökonomische Stabilität, das solide wirtschaftliche Wachstum und die sozialen Fortschritte, die in jüngerer Zeit erzielt werden konnten, wäre Brasiliens aktive internationale Rolle kaum in der zu beobachtenden Form möglich gewesen. Auch wenn trotz eines forcierten Kampfes gegen die Armut nach wie vor große gesellschaftliche Ungleichheit herrscht, auch wenn trotz der Einführung neuer Elemente partizipativer Demokratie nach wie vor grundlegende politische Reformen notwendig sind und auch wenn trotz mehr Transparenz des öffentlichen Sektors die weit verbreitete Korruption nach wie vor ein gravierendes Problem ist, so bildeten die im Lande selbst erzielten Fortschritte doch eine wichtige Voraussetzung dafür, dass sich Brasilien auch international als glaubwürdiger Vertreter demokratischer, partizipativer und sozialer Werte engagieren und globale Strukturreformen einfordern konnte. Sonst wäre es auch kaum möglich gewesen, dass Präsident Lula gleichermaßen bei den Globalisierungskritikern im Rahmen des Weltsozialforums wie bei der in Davos versammelten internationalen Business-Elite als gern gesehener Gast willkommen geheißen wurde.

Das verstärkte außenpolitische Engagement diente nicht zuletzt der Schaffung günstiger Rahmenbedingungen für die internationalen Aktivitäten brasilianischer Unternehmen. Eine Reihe von Großkonzernen investiert in zunehmendem Maße in lateinamerikanische und globale Märkte. Dies war nicht nur wegen der für Brasilien günstigen weltwirtschaftlichen Rahmenbedingungen (enorm gewachsene Nachfrage aus Asien, hohe Rohstoffpreise) möglich, sondern auch, weil einige Unternehmen große Produktivitätsfortschritte erzielt haben und international konkurrenzfähig sind. Sonst wäre der Aufstieg des Landes zu einem der größten Agrarexporteure kaum möglich gewesen. Dadurch änderte sich gleichzeitig die Erwartungshaltung von Teilen der Privatwirtschaft gegenüber der Regierung. War es früher vor allem der defensiv orientierte Schutz des brasilianischen Binnenmarktes, den die Unternehmen von der Regierung einforderten (eine von Teilen der Industrie nach wie vor vertretene Position), so erwartet man heute auch eine aktive Mitgestaltung der internationalen 
Rahmenbedingungen im Sinne der 'nationalen Interessen'. Hier wird einmal mehr der desarrollistische Grundzug der Außenpolitik deutlich.

Brasiliens Aufstieg zum global player ist aber auch auf Veränderungen in Lateinamerika und im internationalen System insgesamt zurückzuführen, auf eine Krisen- und Übergangssituation, aus der sich für Brasilien neue Handlungsspielräume ergaben. Diese Faktoren können an dieser Stelle nicht ausführlich diskutiert werden, sie seien aber zumindest kurz genannt:

- die Krise des neoliberalen Entwicklungsmodells und die Suche nach alternativen Wegen. In Lateinamerika schlug sich diese Tendenz in den Wahlsiegen anti-neoliberaler Parteien und Bewegungen und in der Machtübernahme durch linke Regierungen nieder;

- die Krise des kapitalistischen Akkumulationsmodells, wie sie sich vor allem in der internationalen Finanzkrise nach 2008 zeigte;

- die weitverbreitete Kritik am nach dem Ende des Zweiten Weltkrieges etablierten System von Bretton Woods und der Ruf nach einer Reform des internationalen Finanzsystems;

- die weltweit zunehmenden Forderungen nach einer Reform der Vereinten Nationen;

- die Tendenzen zu einem polyzentrisch strukturierten internationalen System mit zunehmender Machtdiffusion;

- die Krise der US-Hegemonie und das Fehlen einer klar konturierten Lateinamerikapolitik der USA.

Brasilien hat es verstanden, die günstigen internen und externen Rahmenbedingungen zu nutzen, um seiner Außenpolitik ein in dieser Form nie zuvor gekanntes Profil zu verleihen. Ein zentraler Bestandteil dieses Engagements ist eine aktive Süd- bzw. Lateinamerikapolitik.

\section{Brasilien und Lateinamerika}

Brasilien nimmt mehr als die Hälfte des Territoriums Südamerikas ein und weist Grenzen mit 10 der 12 unabhängigen Staaten des Subkontinents auf. Die zentrale geographische Position wird noch akzentuiert durch die Bevölkerungsgröße (fast 200 Mio.) und die Wirtschaftskraft des Landes. Seit den 1990er Jahren und nochmals verstärkt seit den beiden Amtszeiten von 
Präsident Lula bemüht sich Brasilien aktiv um einen Ausbau seiner politischen, ökonomischen und kulturellen Beziehungen mit den Nachbarn in Südamerika. Lange Zeit waren die geographischen Schwerpunkte der Außenpolitik anders definiert: Während des gesamten 19. Jahrhunderts und eines großen Teils des 20. Jahrhunderts strebte Brasilien vor allem gute Beziehungen mit Europa und den Vereinigten Staaten an. Demgegenüber zeichnete sich die Haltung gegenüber Lateinamerika eher durch Desinteresse oder Distanz aus. Dies änderte sich im letzten Drittel des 20. Jahrhunderts aus mehreren Gründen. Erstens wurden die Erwartungen Brasiliens an die "ungeschriebene Allianz" mit den USA immer wieder enttäuscht, weshalb man sich verstärkt um eine Zusammenarbeit mit den südamerikanischen Nachbarn bemühte, zumal sich dafür nach dem Ende des Ost-West-Konfliktes auch neue Spielräume ergaben. Zweitens bot sich nach dem Ende der traditionellen Rivalität mit Argentinien die Möglichkeit, gemeinsam mit dem Nachbarland eine Verbesserung des internationalen Status anzustreben. Drittens erhöhte sich im Zuge der Demokratisierungsprozesse in Südamerika seit den 1980er Jahren nicht nur die Kooperationsbereitschaft der Regierungen, sondern auch der Austausch auf zivilgesellschaftlicher Ebene. Viertens nahm die Bedeutung der südamerikanischen Märkte für die brasilianische Volkswirtschaft zu. Der Anteil der Exporte nach Lateinamerika an den Gesamtexporten des Landes stieg von etwas mehr als $10 \%$ auf ca. ein Viertel. Zudem liegt der Anteil der Industrieprodukte an den Exporten in die Region mit über $90 \%$ so hoch wie gegenüber keiner anderen Weltregion.

Das bilaterale Verhältnis mit dem wichtigsten Nachbarn Argentinien war seit dem 19. Jahrhundert durch Rivalitäten geprägt. Phasen einer engeren Zusammenarbeit wurden immer wieder durch Perioden abgelöst, in denen die Konflikte zwischen den Nachbarländern zunahmen. Lange Zeit hatte Argentinien einen deutlichen Entwicklungsvorsprung vor Brasilien, der aber seit den 1960er Jahren verloren ging. Die Bevölkerung Brasiliens ist mit knapp 200 Mio. Einwohnern heute fast fünfmal so groß wie die Argentiniens, während die Relation noch Mitte des 20. Jahrhunderts in etwa 1 zu 3 (17 Mio. Argentinier, 52 Mio. Brasilianer) betrug. Das Bruttoinlandsprodukt Brasiliens war 2008 etwa viermal so hoch wie das Argentiniens, während beide Länder in den 1960er Jahren noch fast gleichauf lagen. Den seit Mitte der 1970er Jahre in mehreren Schüben erfolgten Desindustrialisierungstendenzen in Argentinien stand ein relativ kontinuierliches Wachstum der brasilianischen Industrieproduktion gegenüber. 
Seit Ende der 1970er Jahre konnten die wechselseitigen Spannungen Schritt für Schritt abgebaut werden. Die Annäherung begann in der Endphase der Militärdiktaturen. 1980 unterzeichneten beide Länder mehrere Kooperationsabkommen im Energie- und Nuklearsektor. Während des Falkland/Malvinen-Konfliktes zwischen Argentinien und Großbritannien im Jahr 1982 zeigte sich Brasilien solidarisch mit Argentinien. Die brasilianische Regierung unterstützte zwar nicht die Anwendung militärischer Gewalt gegenüber Großbritannien, wohl aber den argentinischen Rechtsanspruch auf die Inselgruppe im Südatlantik. Im Zuge des Übergangs zur Demokratie in Argentinien (1983) und Brasilien (1985) verbesserten und intensivierten sich die Beziehungen weiter. 1986 unterzeichneten beide Länder ein Abkommen über die Liberalisierung des bilateralen Handels. 1990 vereinbarten sie die Etablierung einer Wirtschaftsgemeinschaft und luden wenig später auch Uruguay und Paraguay ein, sich an der Initiative zu beteiligen, die im März 1991 in die Gründung des Gemeinsamen Marktes des Südens (Mercosul) mündete. Die wirtschaftliche Zusammenarbeit im Rahmen des Mercosul entwickelte sich zunächst zu einer Erfolgsgeschichte. Während der 1990er Jahre wuchs der bilaterale Handel um das Siebenfache. Argentinien wurde zum zweitwichtigsten Abnehmer brasilianischer Produkte, während Brasilien zum wichtigsten Exportmarkt für Argentinien avancierte. Im Bereich Sicherheitspolitik vereinbarten beide Länder wichtige vertrauensbildende Maßnahmen. Mit der Deklaration von Rio im Jahr 1997 definierten sie ihre bilaterale Beziehung als "strategische Allianz".

Gleichwohl erschwerten unterschiedliche außenpolitische Strategien und zunehmende Handelskonflikte eine engere Zusammenarbeit. Argentinien, das die langjährige "ungeschriebene Allianz" zwischen Brasilien und den USA immer mit Skepsis und Misstrauen verfolgt hatte, bemühte sich während der Regierungszeit von Präsident Menem (1989-1999) selbst um eine Sonderbeziehung zu den USA. Damit rückte Brasilien als außenpolitischer Partner in die zweite Reihe, auch wenn es als Handelsund Wirtschaftspartner wichtig blieb. Mit Nestor Kirchner (2003-2007) und Luiz Inácio Lula da Silva (2003-2010) kamen zwar in beiden Ländern Präsidenten an die Regierung, die dem Mercosul grundsätzlich positiv und der Liberalisierungspolitik der neunziger Jahre eher skeptisch gegenüberstanden. Trotzdem gelang es nicht, der subregionalen Wirtschaftsintegration neuen Schwung zu verleihen. Mehr als 20 Jahre nach Gründung des Mercosul ist nur ein geringer Teil der ursprünglichen Ziele verwirklicht 
worden. Die Perfektionierung der Zollunion ist in weite Ferne gerückt, vielmehr häufen sich die Handelskonflikte zwischen den Mitgliedsstaaten. Selbst das Funktionieren als Freihandelszone ist angesichts immer neuer nicht-tarifärer Handelshemmnisse und Quoten für den Handel in sensiblen Bereichen nicht mehr gewährleistet. Weniger als die Hälfte der zwischen den Mitgliedsstaaten vereinbarten gemeinsamen Regeln, Normen und Standards wurde in nationale Regelungen überführt. Der Mercosul verfügt über eine komplexe institutionelle Struktur, die allerdings einer rein intergouvernementalen Logik folgt. Die Etablierung von supranationalen Steuerungsmechanismen stößt auf entschiedenen Widerstand. Entscheidungsprozesse sind in den nationalen Exekutiven konzentriert. Es ist nicht gelungen, gemeinsame Visionen zu entwickeln, und insgesamt dominiert der Eindruck der Fragmentation. Die kleinen Mitgliedsländer Paraguay und Uruguay sehen sich als Integrationsverlierer und erwarten mehr Unterstützung und größere Anstrengungen zum Abbau der innergemeinschaftlichen Asymmetrien. Die Einrichtung eines Strukturellen Konvergenzfonds (FOCEM) im Jahr 2007 war zwar ein Schritt in die richtige Richtung, sein finanzieller Umfang ist mit ca. 125 Mio. US-Dollar jedoch eher bescheiden. Auch wenn Brasilien 70 \% der Beiträge zu dem Fonds beisteuert, scheint es stark übertrieben, das Land deswegen in Anlehnung an die langjährige deutsche Rolle in Europa bereits als Zahlmeister des Integrationsprozesses zu bezeichnen, wie dies verschiedene Experten in jüngerer Zeit getan haben. Obwohl die Regierungen aller Mitgliedsstaaten in der Vergangenheit wiederholt von einer "Vertiefung" und "Wiederankurbelung" des Mercosul gesprochen haben, scheint der politische Wille dazu nicht vorhanden zu sein. Zwar ist aufgrund der damit verbundenen politischen Kosten nicht damit zu rechnen, dass eines der Mitgliedsländer das Bündnis verlässt, aber vor allem Brasilien scheint nicht daran interessiert zu sein, dem Mercosul größere Entscheidungskompetenzen zuzugestehen. Die strukturellen Asymmetrien zwischen Brasilien und den anderen Mitgliedsländern haben sich seit Gründung des Mercosul eher verstärkt als reduziert. Argentinien hat für Brasilien stark an Bedeutung verloren. Noch Anfang der 1990er Jahre war Argentinien für Brasilien als Energie- und Lebensmittellieferant nicht unwichtig. Inzwischen ist Brasilien selbst in beiden Bereichen zu einem Exporteur geworden. Die wiederholten wirtschaftlichen und politischen Krisen in Argentinien bei einer gleichzeitig relativ kontinuierlichen und erfolgreichen Entwicklung Brasiliens haben die 'strategische Partnerschaft' zwischen beiden Ländern stark 
relativiert. Brasilien ist trotz Integrationsrhetorik nicht dazu bereit, seinem Streben nach außenpolitischer Autonomie und Universalismus durch eine institutionelle Stärkung des Mercosul Schranken setzen zu lassen.

Parallel zur subregionalen Wirtschaftsintegration im Rahmen des Mercosul ergriffen die brasilianischen Regierungen seit den 1990er Jahren weitere Initiativen zum Ausbau der regionalen Zusammenarbeit. Im Jahr 1992 vereinbarten die Unterzeichner des Amazonaspaktes von 1978 (Bolivien, Brasilien, Kolumbien, Ecuador, Guyana, Peru, Surinam und Venezuela) eine sogenannte Amazonasinitiative zur engeren Zusammenarbeit im Bereich der physischen Infrastruktur. 1994 schlug die brasilianische Regierung die Errichtung einer Südamerikanischen Freihandelszone (ALCSA) vor, die allerdings ebenso wenig verwirklicht wurde wie der von den USA im gleichen Jahr lancierte Vorschlag einer Gesamtamerikanischen Freihandelszone (Área de Libre Comercio de las Américas; ALCA). Im September 2000 lud der damalige Präsident Cardoso erstmals alle südamerikanischen Staats- und Regierungschefs zu einem Gipfeltreffen in die brasilianische Hauptstadt Brasília ein. Das Treffen gilt als Geburtsstunde der "Initiative zur regionalen Infrastrukturintegration in Südamerika" (IIRSA), mit der die physische Integration Südamerikas vorangetrieben werden sollte. Beim dritten südamerikanischen Gipfeltreffen im peruanischen Cusco wurde 2004 die Gemeinschaft Südamerikanischer Nationen (CSN) ins Leben gerufen, die 2007 auf Vorschlag Venezuelas den Namen Union Südamerikanischer Nationen (UNASUL) erhielt. Zentrales Ziel der UNASUL ist die Stärkung der kulturellen, sozialen, wirtschaftlichen und politischen Zusammenarbeit in Südamerika. Der im Mai 2008 unterzeichnete Gründungsvertrag wurde von allen zwölf unabhängigen Staaten Südamerikas unterzeichnet. Ebenfalls aufgrund einer brasilianischen Initiative entstand Ende 2008 im Rahmen der UNASUL der Südamerikanische Verteidigungsrat.

Die brasilianische Diplomatie sprach seit den 1990er Jahren immer öfter von Südamerika und seltener von Lateinamerika, wenn es um die Definition der eigenen regionalen Zugehörigkeit ging. Dies war kein Zufall, sondern bewusste Strategie. Dahinter steckte insbesondere die Ansicht, dass Mexiko sich aufgrund seiner starken Hinwendung zu den USA und spätestens mit Unterzeichnung des Nordamerikanischen Freihandelsabkommens (NAFTA) im Jahr 1994 so weit von Südamerika entfernt habe, dass es keinen Sinn mehr mache, von Lateinamerika zu sprechen. Mexiko, Zentralamerika und die Karibik wurden dem geopolitischen Ein- 
flussgebiet der USA zugerechnet, während Südamerika als eine natürliche Interessensphäre Brasiliens angesehen wurde. Gleichwohl ist die Bezugnahme auf Lateinamerika nicht vollständig von der diplomatischen Agenda Brasiliens verschwunden, im Gegenteil, in den vergangenen Jahren ist wieder öfter die Rede davon. Im Dezember 2008 lud Präsident Lula die Staats- und Regierungschefs aller Länder Lateinamerikas und der Karibik zu einem Gipfeltreffen nach Costa do Sauípe ein, um über Integrationsund Entwicklungsfragen zu sprechen. 33 Länder, einschließlich Kuba, waren bei dem Treffen anwesend. Zum ersten Mal in 200 Jahren unabhängiger Geschichte - so betonten die brasilianischen Gastgeber - hatten sich damit die lateinamerikanischen und karibischen Länder ohne externe Bevormundung versammelt. Beim zweiten CALC-Gipfeltreffen an der mexikanischen Riviera Maya im Februar 2010 wurde die Gründung einer Gemeinschaft der lateinamerikanischen und karibischen Staaten (CELAC) beschlossen, die dann im Dezember 2011 in Caracas erfolgte.

Brasilien war nicht das einzige Land, das im vergangenen Jahrzehnt Impulse für einen Ausbau der regionalen Zusammenarbeit gab. Insbesondere Venezuela trat unter Präsident Hugo Chávez durch entsprechende Initiativen hervor. Mit der Bolivarischen Allianz für die Völker Amerikas (ALBA), einem auf den Grundprinzipien Kooperation, Solidarität, Komplementarität und Selbstbestimmung basierenden alternativen Integrationsmodell, sowie mit zahlreichen Initiativen für eine verstärkte regionale Zusammenarbeit in den Bereichen Militär, Finanzen, Umwelt, Bildung, Wissenschaft und Technologie, Gesundheit und Medien gelang es dem im März 2013 verstorbenen Chávez, zum charismatischen Anführer einer gegen die die kapitalistische Globalisierung und den 'US-Imperialismus' gerichteten Bewegung zu werden. Obwohl Brasilien mit dem von Chávez propagierten bolivarischen Mythos einer lateinamerikanischen Einheit weder in historischer Perspektive noch im Hinblick auf die damit verbundenen aktuellen Zielsetzungen übereinstimmt, bemühten sich die Regierungen Lula und Rousseff immer darum, Venezuela nicht auszugrenzen und die von den beiden Ländern propagierten Integrationsmodelle als komplementär darzustellen. Die auf Provokation und radikalen Positionen basierende venezolanische Politik hatte für Brasilien zudem den Vorteil, dass dadurch die eigene Glaubwürdigkeit als Garant einer zwar progressiven, aber gleichzeitig moderaten und vermittelnden Position zusätzlich gestärkt wurde. Diese vermittelnde Rolle Brasiliens wurde auch durch das Engagement der brasilianischen Diplomatie bei der Krisenbewältigung 
in und zwischen Nachbarländern, beispielsweise beim Konflikt zwischen Peru und Ecuador (1995), in Paraguay (1996), in Haiti und Bolivien sowie im Hinblick auf die angespannte Situation in Venezuela deutlich. Im Jahr 2004 übernahm Brasilien zudem die Führung der VN-Friedensmission in Haiti.

Trotz dieser vielfältigen politischen Initiativen tut sich Brasilien nach wie vor schwer mit einer Führungsrolle in Südamerika. Dies hängt zum einen damit zusammen, dass sich die brasilianische Außenpolitik stets im Spannungsfeld zwischen erwünschter Übernahme von Verantwortung einerseits und Ängsten der Nachbarn vor einem brasilianischen Hegemoniestreben andererseits bewegen muss. Zudem hat Brasilien zwar ansatzweise damit begonnen, finanzielle Kosten des regionalen Integrationsprozesses zu übernehmen, es ist aber nicht dazu bereit, eine Stärkung subregionaler oder regionaler Entscheidungsinstanzen auf Kosten der eigenen auBenpolitischen Entscheidungsfreiheit zu akzeptieren. In wirtschaftlicher Hinsicht wird eine brasilianische Führungsrolle in Südamerika auch durch die starken entwicklungsstrategischen Interessendivergenzen zwischen den gegenwärtigen Regierungen erschwert. Während Länder wie Chile, Peru und Kolumbien sich auf der Grundlage bi- und multilateraler Freihandelsabkommen zunehmend in Richtung pazifisches Asien orientieren, vertreten Länder wie Venezuela, Bolivien und Ecuador extrem globalisierungskritische Positionen. In entwicklungsstrategischer Hinsicht nimmt Brasilien eine Zwischenposition ein, es ist jedoch für keines seiner Nachbarländer ein entwicklungsstrategisches Modell. Der Schutz der nationalen Industrie war und ist ein zentrales Ziel der Außen- und Außenwirtschaftspolitik. Brasilien verfügt heute über den größten und am stärksten diversifizierten Industriesektor der Region, darunter sind aber auch zahlreiche wenig wettbewerbsfähige Sektoren. Der große brasilianische Binnenmarkt könnte ein wichtiger Anziehungspunkt für die Nachbarländer sein, er gilt jedoch als hochgradig geschützt. Brasiliens staatliche Entwicklungsbank BNDES ist zwar inzwischen zu einer der kapitalstärksten Entwicklungsbanken weltweit avanciert und engagiert sich in zunehmendem Maße auch in großen Infrastrukturprojekten außerhalb Brasiliens, die zur Verfügung gestellten Mittel kommen jedoch ausschließlich brasilianischen Unternehmen zugute. Die Bank ist damit kein Instrument einer regionalen Konvergenzpolitik, sondern eher ein weiteres Instrument im Arsenal der brasilianischen Wirtschaft, um ihre führende Rolle in Südamerika auszubauen. 


\section{Die Süd-Süd-Politik}

Neben den innerlateinamerikanischen Beziehungen ist zumindest in rhetorischer Hinsicht die Süd-Süd-Politik seit der Regierung Lula zu einem Schwerpunkt der brasilianischen Außenpolitik avanciert. Dazu gehört erstens eine aktive Politik gegenüber dem afrikanischen Kontinent, die sich in der Eröffnung zahlreicher neuer Botschaften, in einer regen Besuchsdiplomatie sowie in einem Ausbau der brasilianischen Entwicklungszusammenarbeit mit afrikanischen Ländern niedergeschlagen hat (ausführlicher Seibert 2009, Zilla/Harig 2012). Zweitens hat Brasilien eine Reihe von Süd-Süd-Gipfeltreffen initiiert, beispielsweise zwischen Südamerika und Afrika, zwischen Südamerika und den arabischen Staaten sowie zwischen Südamerika und Ostasien. Drittens stützt sich die brasilianische Diplomatie in wachsendem Maße auf neue Netzwerke und Allianzen mit anderen aufstrebenden Mächten außerhalb der OECD-Welt. Dabei handelt es sich um Bündnisse mit variabler Geometrie, die nicht notwendigerweise auf gemeinsamen Werten und allgemeinen Prinzipien basieren, sondern je nach konkretem Thema ad hoc zustande kommen (Flemes/Scholvin/Strüver 2011). Die wichtigsten dieser multilateralen Foren sind die BRICS-Gruppe (Brasilien, Russland, Indien, China und Südafrika) und das IBSA-Forum (Indien, Brasilien, Südafrika). Die Bündnisse gelten aus brasilianischer Perspektive als funktional für die Vertretung nationaler Interessen auf globaler Ebene, eine grundsätzliche Schwäche besteht jedoch darin, dass sie alle stark vom schwankenden politischen Willen der beteiligten Länder abhängig sind, der Zusammenarbeit im Rahmen der jeweiligen Außen- und Außenwirtschaftsstrategien einen wichtigen Platz einzuräumen.

Mit der Süd-Süd-Politik verbinden sich für die brasilianische Außenpolitik mehrere Zielsetzungen. Es geht zum einen darum, die eigene Gestaltungsmacht im internationalen System weiter auszubauen und Reformen voranzutreiben, die einen Machtzugewinn für Brasilien bedeuten würden. An erster Stelle ist hier sicherlich das Streben nach einem ständigen Sitz im Sicherheitsrat der VN zu nennen. Es geht zum anderen darum, Entscheidungen und Entwicklungen zu verhindern, die nicht im brasilianischen Interesse liegen. Diese Vetomacht wurde 2003 sehr deutlich, als Brasilien eine führende Rolle im Rahmen der G20 spielte, einer Koalition von Entwicklungs- und Schwellenländern, die mit ihrer entschlossenen Haltung die Verhandlungen im Rahmen der Konferenz der Welthandelsorganisa- 
tion (WTO) in Cancún zum Scheitern brachte, weil die von den USA und der EU vorgelegten Vorschläge als inakzeptabel betrachtet wurden. Nicht zuletzt ist die Süd-Süd-Politik auch ein Instrument, um nach Anerkennung und Statuszugewinnen, sowohl von Seiten der etablierten Mächte als auch von Seiten des Südens, zu streben.

\section{Die bilateralen Beziehungen zu den USA}

Auch wenn der kulturelle, politische und wirtschaftliche Einfluss der USA in Südamerika nie ein vergleichbares Ausmaß erreichte wie in Mexiko, Zentralamerika und der Karibik, so waren und sind die USA doch ein wichtiger Referenzpunkt für die internationale Einbindung Brasiliens. Die Beziehungen zwischen den beiden Ländern haben im 20. Jahrhundert sehr unterschiedliche Phasen durchlaufen, die von der Allianz bis zur wechselseitigen Distanzierung reichten, dabei aber nie in offene Feindschaft umschlugen. Seit der Amtszeit von Außenminister Rio Branco (1902-1912) bemühte sich Brasilien um ein konfliktfreies Verhältnis zu den Vereinigten Staaten. Ein gutes Verhältnis zur Hegemonialmacht im Norden schien nicht nur aufgrund der handelspolitischen Abhängigkeit sinnvoll. Brasilien baute auch darauf, dass eine pragmatische Unterstützung nordamerikanischer Positionen auf hemisphärischer und globaler Ebene im Gegenzug zu einer wohlwollenden Haltung der USA gegenüber brasilianischen Interessen führen und somit die internationale Position Brasiliens stärken würde. Ob diese frühe Form des bandwagoning tatsächlich zu den gewünschten Ergebnissen führte, blieb in Brasilien immer umstritten.

Auch nach dem Zweiten Weltkrieg waren die brasilianischen Erwartungen gegenüber den USA groß: Brasilien hatte den Achsenmächten 1942 den Krieg erklärt und 1944 ein 25.000 Mann starkes Expeditionskorps nach Europa geschickt. Als Dank dafür rechnete man mit wirtschaftlichem und politischem Entgegenkommen der USA. Umso größer war die Enttäuschung darüber, dass Brasilien nicht den erhofften ständigen Sitz im Sicherheitsrat der Vereinten Nationen erhielt. Auch die wirtschaftliche Unterstützung der USA blieb weit hinter den brasilianischen Erwartungen zurück. Der ideologische Konsens der brasilianischen Eliten mit den USA im Kalten Krieg trat daher in dem Maße in den Hintergrund, wie sich in Brasilien ein wirtschaftlicher Nationalismus ausbreitete und Außenpolitik immer mehr als Außenwirtschaftspolitik im Dienste der nationalen Ent- 
wicklung gesehen wurde. Ab Ende der 1960er Jahre wuchs die Unzufriedenheit gegenüber den USA, da diese zunehmend als Garant einer als ungerecht empfundenen Weltwirtschaftsordnung wahrgenommen wurden. In den 1980er Jahren war Brasilien aufgrund wachsender politischer und ökonomischer Konflikte immer weniger dazu bereit, die nordamerikanischen Positionen im Ost-West-Konflikt zu unterstützen. Der bilaterale Handel nahm zwar zu, aber unterschiedliche Auffassungen im Hinblick auf Themen wie die Schuldenkrise, Technologietransfer, den Schutz von Patenten und geistigem Eigentum, den wechselseitigen Marktzugang und Exportsubventionen sorgten für zahlreiche Divergenzen. Dies änderte sich vorübergehend, als Präsident Collor de Mello (1990-1992) eine Öffnung, Deregulierung und Liberalisierung des brasilianischen Binnenmarktes in Gang setzte.

Im Verlauf der 1990er Jahre verdoppelte sich das Volumen des bilateralen Handels und die Direktinvestitionen der USA in Brasilien verdreifachten sich. Die Regierungen Clinton (1993-2001) und Cardoso (1995-2002) arbeiteten in vielen Bereichen zusammen, die bilaterale Agenda blieb jedoch durch eine Reihe von Handelskonflikten belastet. Zum zentralen Thema entwickelten sich dabei die letztlich gescheiterten Verhandlungen über die von den USA 1994 vorgeschlagene Bildung einer Gesamtamerikanischen Freihandelszone (ALCA). Die unterschiedlichen Auffassungen dahingehend, welche Märkte in welchem Ausmaß und zu welchem Zeitpunkt geöffnet werden sollten, konnten nie überwunden werden. Während die USA eine Liberalisierung der Märkte für Industriegüter und Dienstleistungen anstrebten, forderte Brasilien eine Öffnung der Agrarmärkte und einen Abbau von Subventionen und protektionistischen Maßnahmen in den Industrieländern. Außerdem bestand das Land auf einer differenzierten Marktöffnung je nach Größe und Wettbewerbsfähigkeit der beteiligten Volkswirtschaften. Entsprechend dieser Konfliktlinien saßen sich Brasilien und die USA auch bei den Welthandelsgesprächen im Rahmen der WTO in unterschiedlichen Lagern gegenüber. Der Amtsantritt von US-Präsident George W. Bush (2001-2008) und dessen Außenpolitik (Krieg in Afghanistan, Bush-Doktrin, Irak-Invasion) führten zu einer deutlichen Abkühlung des bilateralen Verhältnisses, da Brasilien dieser Politik sehr kritisch gegenüberstand und sich weigerte, Bushs "Koalition der Willigen" beizutreten. Viele Beobachter rechneten daher mit wachsenden Konfrontationen zwischen beiden Ländern, als Präsident Lula 2003 sein Amt antrat. Dazu kam es jedoch nicht. Lula und Bush 
pflegten regelmäßige Kontakte auf höchster Ebene und auch ihre Außenminister standen in einem engen Austausch miteinander. Die bilateralen Beziehungen blieben freundlich, auch wenn in der Sache zum Teil tiefgreifende Interessenunterschiede und Meinungsverschiedenheiten existierten.

Ein zentrales Thema in den Beziehungen zwischen beiden Ländern ist der Handel. Auch wenn auf die USA heute nur noch $14 \%$ der brasilianischen Exporte und $15 \%$ der Importe entfallen, so bleibt der US-Markt doch wichtig für Brasilien, vor allem für Fertiggüterexporte. Einige brasilianische Exportprodukte sind allerdings in den USA mit hohen Einfuhrzöllen und/oder Antidumping-Maßnahmen konfrontiert, beispielsweise Zucker, Tabak, Ethanol und Orangensaft. Beide Länder haben wegen diverser Handelsdispute immer wieder die Streitschlichtungsinstanzen der WTO bemüht. Umgekehrt beklagen die USA hohe brasilianische Zölle für Industriegüter und erwarten mehr Entgegenkommen beim Handel mit Dienstleistungen, Investitionen, Eigentumsrechten und staatlichen Ausschreibungen. Es sind diese grundsätzlichen Fragen, die sich nicht nur mit Blick auf den bilateralen Handel stellen, sondern genauso hinsichtlich der zukünftigen Wirtschafts- und Handelsbeziehungen in den Amerikas und auf globaler Ebene.

Zu einem wichtigen Thema zwischen beiden Ländern sind in den vergangenen Jahren Energiefragen geworden. Dabei geht es zum einen um Biokraftstoffe. Brasilien und die USA haben als die beiden weltgrößten Produzenten von Ethanol im Jahr 2007 ein Memorandum of Understanding unterzeichnet, um die Ethanolproduktion in der westlichen Hemisphäre zu steigern, globale Produktionsstandards in diesem Bereich zu etablieren, Entwicklungs- und Forschungskapazitäten auszubauen und Technologien auszutauschen. Einem weiteren Ausbau der bilateralen Zusammenarbeit steht jedoch unter anderem entgegen, dass brasilianisches Ethanol in den USA mit hohen Einfuhrzöllen belegt wird, während die USA die eigene Ethanolproduktion subventionieren und zugleich den zollfreien Import von Ethanol aus der Karibik und Zentralamerika ermöglichen. Zu Verstimmungen führte wiederholt auch das Thema Atomenergie. Brasilien ist zwar 1998 unter Präsident Cardoso dem Atomwaffensperrvertrag beigetreten, es besteht jedoch auf dem Recht, selbst zu zivilen Zwecken Uran anzureichern.

In politischer Hinsicht begrüßten es die USA offiziell, dass Brasilien seit den 1990er Jahren eine aktivere Rolle in Südamerika übernommen und wiederholt zur Lösung von zwischenstaatlichen Konflikten in der Re- 
gion beigetragen hat. Gleichwohl zeigten sich zwischen dem 'Stabilitätsanker Brasilien' und den USA wachsende Divergenzen im Hinblick auf die Einschätzung regionaler Entwicklungen und die Rolle der USA in der Hemisphäre. Je mehr Brasilien sich zudem um eine Profilierung auf globaler Ebene bemüht und dabei auf einem von den USA unabhängigen Profil besteht, desto eher sind in Zukunft bilaterale Konflikte zu erwarten. Ein klares Beispiel dafür war die heftige Kritik der USA an dem von Brasilien und der Türkei in Verhandlungen mit dem iranischen Präsidenten im Mai 2010 erzielten Kompromiss im Streit um das Atomprogramm des Iran.

\section{Die Beziehungen mit der Europäischen Union}

Neben den bilateralen Beziehungen mit wichtigen europäischen Partnern wie Frankreich, Deutschland und Spanien konzentriert sich die brasilianische Politik gegenüber Europa vor allem auf vier Bereiche: den Handel, die Verhandlungen über ein Assoziierungsabkommen zwischen dem Mercosul und der EU, die 2007 abgeschlossene strategische Partnerschaft mit der EU sowie die Zusammenarbeit zur Gestaltung globaler Strukturen und Prozesse. Mit einem Außenhandelsvolumen von fast 77 Mrd. Euro (2012) ist die EU einer der wichtigsten Handelspartner Brasiliens. Brasilianischen Exporten im Wert von 37,1 Mrd. Euro standen dabei Importe im Wert von 39,6 Mrd. Euro entgegen. Für Brasilien ist die EU der wichtigste Abnehmer von landwirtschaftlichen Produkten wie Zucker, Sojabohnen und Rindfleisch. Gleichwohl sind gerade die Handelsbeziehungen immer wieder zur Quelle von Konflikten geworden, da hier unterschiedliche Interessen aufeinandertreffen. Während Brasilien von der EU seit langem einen verbesserten Marktzugang für seine landwirtschaftlichen Produkte (und in den vergangenen Jahren auch für Biotreibstoff) fordert und die Agrarsubventionen der EU kritisiert, steht die EU den hohen Zugangshürden zum brasilianischen Markt für Industriegüter und Dienstleistungen skeptisch gegenüber und fordert einen verbesserten Schutz von geistigem Eigentum. Diese Divergenzen haben bislang auch den erfolgreichen Abschluss eines Assoziierungsabkommens zwischen dem Mercosul und der EU verhindert, über das seit 1999 verhandelt wird und das eine breite Palette an Themen umfassen sollte, vom politischen Dialog über den Handel mit Agrargütern und Dienstleistungen bis hin zu Regeln für öffentliche Beschaffungen, Investitionen und geistige Eigentumsrechte. Obwohl 
beide Seiten wiederholt ihr Interesse an einem solchen Abkommen beteuert haben, scheint letztlich der politische Wille zu fehlen, um sich auf die dafür notwendigen Kompromisse einzulassen.

Durch die 2007 vereinbarte strategische Partnerschaft zwischen der EU und Brasilien sollte die Zusammenarbeit in Energiefragen, im Hinblick auf die Bekämpfung des Klimawandels und in puncto Gestaltung der globalen Strukturpolitik intensiviert werden. Doch obwohl sich die Häufigkeit der Treffen seitdem erhöht hat, ist es nicht gelungen, substanzielle Kompromisse zu erzielen. Auch wenn beide Seiten immer wieder ihre gemeinsamen Grundwerte und die Präferenz für multilaterale Strategien betonen, so liegen die konkreten Vorstellungen im Hinblick auf die Gestaltung des internationalen Systems doch oft weit auseinander. Hinzu kommt, dass Europa aus brasilianischer Perspektive zunehmend als 'Kontinent der Vergangenheit' angesehen wird. Die EU, so der brasilianische Sicherheitsexperte Alcides Costa Vaz, sei jahrhundertelang eine Kernregion und ein führender Akteur der Weltpolitik gewesen. Wirtschaftliche, geostrategische und demographische Entwicklungen hätten jedoch - verstärkt nach dem Ende des Ost-Welt-Konfliktes - dazu geführt, dass diese Rolle zunehmend in Frage gestellt werde. Die EU sei nach wie vor eine Exportmacht, ein wichtiger Akteur in multilateralen Institutionen und der engste Verbündete der USA, aber ihre relative Macht und ihr internationaler Einfluss würden als stagnierend oder sogar als zurückgehend wahrgenommen. Demgegenüber spielten neue Akteure wie Brasilien eine zunehmende Rolle auf globaler Ebene (Costa Vaz 2013). Solche Aussagen zeigen nicht nur das gewachsene Selbstbewusstsein Brasiliens, sondern sie erklären auch, warum Brasilien nur bedingt dazu bereit ist, sich auf Kompromisse mit einer EU - zumal einer durch die EuroKrise noch verstärkt in Mitleidenschaft gezogenen EU - einzulassen, von der man annimmt, dass sie ohnehin in Zukunft eine immer geringere Rolle spielen wird.

\section{Fazit}

Brasilien ist in den vergangen zwei Jahrzehnten zu einem internationalen Akteur ersten Ranges avanciert. Dazu beigetragen haben sowohl die im Inneren des Landes erzielten politischen, wirtschaftlichen und sozialen Erfolge als auch die veränderten internationalen Rahmenbedingungen. 
Die brasilianische Diplomatie hat es verstanden, dieses Umfeld geschickt zu nutzen, um sich nicht nur in der eigenen Region Süd- bzw. Lateinamerika, sondern auch auf globaler Ebene als wichtiger politischer Akteur zu etablieren. Die Professionalität und die langfristige Orientierung der brasilianischen Außenpolitik machen das Land zu einem zuverlässigen Partner. Gleichwohl ist Brasilien nicht nur ein zäher Verhandlungspartner, sondern das internationale Engagement des Landes folgt auch nahezu ausschließlich einem strikten Kalkül nationalen Interesses und ist stets darauf bedacht, auf keinen Fall eine Einschränkung der nationalen Autonomie zuzulassen. Dies zeigt sich nicht nur im Rahmen der regionalen Kooperations- und Integrationsprozesse in Lateinamerika, sondern auch in den anderen Bereichen der brasilianischen Außenpolitik.

\section{Literaturverzeichnis}

Amorim, Celso (2010): "Brazilian Foreign Policy under President Lula (2003-2010): An Overview”. In: Revista Brasileira de Política Internacional, 53 (Special Edition), 214-240.

BiRLE, Peter (2006a): "Brasilien und die Amerikas: Lateinamerika und die USA als Bezugspunkte der brasilianischen Außenpolitik". In: Birle, Peter et al. (Hg.): Hemisphärische Konstruktionen der Amerikas. Frankfurt am Main: Vervuert, 139-166.

— (2006b): "Von Rivalen zu strategischen Partnern? Selbst- und Fremdbilder in den bilateralen Beziehungen zwischen Argentinien und Brasilien". In: Birle, Peter/Nolte, Detlef/Sangmeister, Hartmut (Hg.): Demokratie und Entwicklung in Lateinamerika. Frankfurt am Main: Vervuert, 311-339.

- (2009): "Zwischenstaatliche Konflikte in Südamerika vom 19. Jahrhundert bis heute. Ursachen, Lösungsansätze, Perspektiven”. In: Mark, Lothar/Fritz, Erich G. (Hg.): Lateinamerika im Aufbruch. Eine kritische Analyse. Oberhausen: Athena, 123-136.

- (2010a): "Argentinien und Brasilien zwischen Rivalität und Partnerschaft". In: Aus Politik. und Zeitgeschichte, 12/2010, 3-8.

- (2010b): "Zwischen Integration und Fragmentierung: Regionale Zusammenarbeit in Lateinamerika". In: Birle, Peter (Hg.): Lateinamerika im Wandel. Baden-Baden: Nomos, $75-98$.

Burges, Sean W. (2008): “Consensual Hegemony: Theorizing Brazilian Foreign Policy after the Cold War". In: International Relations, 22, 1, 65-84.

Costa Vaz, Alcides (2013): Brazilian Perspectives on the Changing Global Order and Security Challenges. Bruxelles: Center for European Policy Studies (CEPS Working Document No. 376).

Daudelin, Jean (2007): “Joining the Club: Lula and the End of Periphery for Brazil”. In: Birle, Peter/Costa, Sérgio/Nitschack, Horst (Hg.): Brazil in the Americas. Convergences and Perspectives. Madrid/Frankfurt am Main: Iberoamericana/Vervuert, 51-77. 


\section{$168 \mid$ Peter Birle}

Flemes, Daniel/Scholvin, Sören/Strüver, Georg (2011): "Aufstieg der Netzwerkmächte". In: GIGA Focus Global, 2, 1-8.

GrabendorfF, Wolf (2013): "Brasilien auf dem Weg zur Weltmacht?" In: Der Bürger im Staat, 1-2, 117-124.

Gratius, Susanne (2012): Brazil and the European Union: Between Balancing and Bandwagoning. Madrid: Egmont Institute/FRIDE.

Hirst, Monica (2005): The United States and Brazil: a long Road of Unmet Expectations. New York: Routledge.

LAFer, Celso (2001): A identidade internacional do Brasil e a politica externa brasileira. Passado, presente e futuro. São Paulo: Perspectiva.

Malamud, Andrés (2011): "A Leader without Followers? The Growing Divergence Between the Regional and Global Performance of Brazilian Foreign Policy". In: Latin American Politics and Society, 3, 1-24.

Moniz BandeIra, Alberto Luiz (2006): "Brazil as a Regional Power and its Relations with the United States". In: Latin American Perspectives, 148, 12-27.

Meimann, Kellie/Rothkopf, David (2009): The United States and Brazil. Two Perspectives on Dealing with Partnership and Rivalry. Washington, DC: Center for American Progress.

Pastrana Buelvas, Eduardo/Jost, Stefan/Flemes, Daniel (Hg.) (2012): Colombia y Brasil: ¿socios estratégicos en la construcción de Suramérica? Bogotá: Editorial Pontificia Universidad Javeriana.

Rousseff, Dilma (2013): “Discurso da Presidenta da República, Dilma Rousseff, durante cerimônia de formatura da turma 2011/2013 do Instituto Rio Branco. Palácio Itamaraty, 17 de junho de 2013". <http://www.itamaraty.gov.br/> (3.7.2013).

SEIBERT, Gerhard (2009): "Brasilien in Afrika: Globaler Geltungsanspruch und Rohstoffe". In: GIGA Focus Africa, 8, 1-8.

Sorj, Bernardo/Fausto, Sergio (Hg.) (2011): Brasily América del Sur. Miradas Cruzadas. Buenos Aires: Catálogos.

- (2013): Brasily América Latina: ¿Qué Liderazgo es Posible? Rio de Janeiro: Plataforma Democrática.

Vigevani, Tullo/Cepaluni, Gabriel (2010): Brazilian Foreign Policy in Changing Times. The Quest for Autonomy from Sarney to Lula. Lanham: Lexington Books.

Zilla, Claudia (2011): Brasilianische Außenpolitik. Nationale Tradition, Lulas Erbe und Dilmas Optionen. Berlin: Stiftung Wissenschaft und Politik.

Zilla, Claudia/Harig, Christoph (2012): Brasilien als emerging donor: politische Distanz und operative Nähe zu den traditionellen Gebern. Berlin: Stiftung Wissenschaft und Politik. 